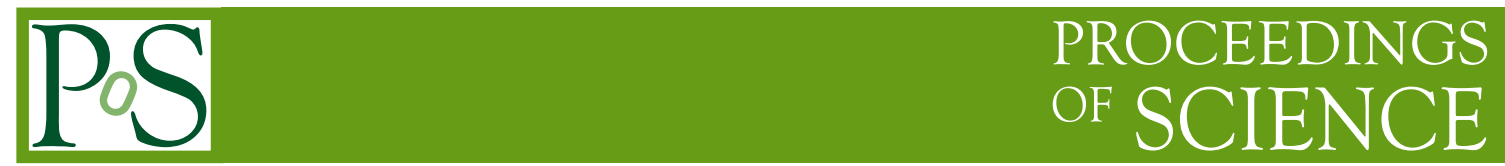

\title{
Testing Quantum Electrodynamics with Positronium State
}

\author{
Alexander A. Penin ${ }^{* a b}$ \\ ${ }^{a}$ Department of Physics, University of Alberta, \\ Edmonton AB T6G 2J1, Canada \\ ${ }^{b}$ Institut für Theoretische Teilchenphysik, Karlsruhe Institute of Technology (KIT), \\ 76128 Karlsruhe, Germany \\ E-mail: penineualberta.ca
}

In this proccedings I report the complete result for the $\mathscr{O}\left(\alpha^{7} m_{e}\right)$ one-photon annihilation contribution to the hyperfine splitting of the ground state energy levels in positronium [四].

Loops and Legs in Quantum Field Theory - LL 2014,

27 April 2014 - 02 May 2014

Weimar, Germany

\footnotetext{
* Speaker.
} 
Positronium, an electromagnetic bound state of an electron and a positron, is the lightest known atom. The strong interaction effects in positronium are suppressed by the small ratio of the electron mass $m_{e}$ to the hadronic mass scale, and the properties of the bound state can be calculated perturbatively in quantum electrodynamics (QED) as an expansion in Sommerfeld's fine-structure constant $\alpha$, with very high precision only limited by the complexity of the calculations. Positronium is thus a unique laboratory for testing the QED theory of weakly bound systems. At the same time a deviation of the QED predictions from the results of experimental measurements may be a signal of an exotic "new physics" []].

Positronium hyperfine splitting (HFS) is defined by the mass difference between the spintriplet orthopositronium and spin-singlet parapositronium states. Already three decades ago HFS in positronium has been determined with the precision of about ten parts in a million [3], 团] yielding

$$
\Delta v^{\exp }=203.3875(16) \mathrm{GHz}
$$

and

$$
\Delta v^{\exp }=203.38910(74) \mathrm{GHz},
$$

respectively. Recently a new result with reduced systematic uncertainty from the positronium thermalization effect has been reported [5]

$$
\Delta v^{\exp }=203.3942(16)_{\text {stat. }}(13)_{\text {syst. }} \mathrm{GHz}
$$

which overshoots the previous measurements by 2.6 standard deviations.

The present theoretical knowledge may be summarized as:

$$
\begin{aligned}
\Delta v^{\text {th }} & =\Delta v^{L O}\left\{1-\frac{\alpha}{\pi}\left(\frac{32}{21}+\frac{6}{7} \ln 2\right)-\frac{5}{14} \alpha^{2} \ln \alpha+\left(\frac{\alpha}{\pi}\right)^{2}\left[\frac{1367}{378}-\frac{5197}{2016} \pi^{2}+\left(\frac{6}{7}+\frac{221}{84} \pi^{2}\right)\right.\right. \\
& \left.\left.\times \ln 2-\frac{159}{56} \zeta(3)\right]+\left(\frac{\alpha}{\pi}\right)^{3}\left[-\frac{3}{2} \pi^{2} \ln ^{2} \alpha+\left(-\frac{62}{15}+\frac{68}{7} \ln 2\right) \pi^{2} \ln \alpha+D\right]\right\}
\end{aligned}
$$

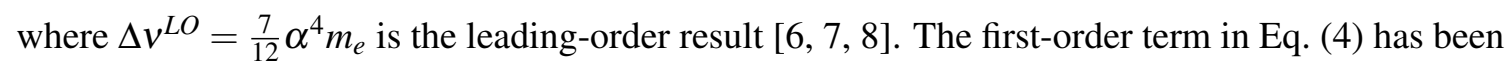
computed in Ref. [Q]. The second-order corrections have been derived by several authors [एய, प1],

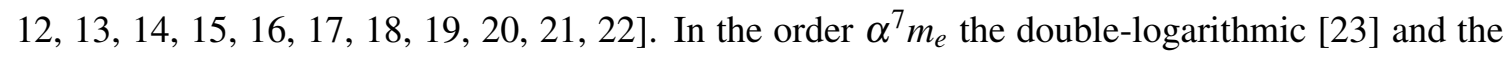
single-logarithmic terms [24, [25, [26] are known, while the nonlogarithmic coefficient $D$ is not yet available. Including all the terms known so far, we have [R6]

$$
\Delta v^{\text {th }}=203.39169(41) \mathrm{GHz}
$$

where the error is estimated by the size of the third-order nonlogarithmic contribution to the HFS in muonium atom [2]], which however does not include annihilation and recoil effects. The result (I)

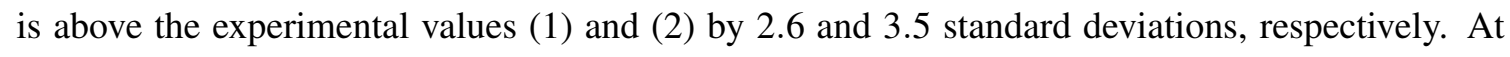
the same time, it is only 1.2 standard deviations below the most recent result (B]). Thus the status of the QED prediction for positronium HFS remains ambiguous.

Much activity is currently under way to improve the experimental precision [ [28, [2]]. On the theoretical side the accuracy is limited by the unknown third-order coefficient $D$. The corresponding uncertainty may soon become a limiting factor in the study of positronium HFS and the 
calculation of the nonlogarithmic third-order term in Eq. (\$) would be timely. This calculation, however, is an extremely challenging problem of perturbative quantum field theory complicated by the presence of multiple scales and bound-state dynamics.

The first major step towards the solution of this problem has been done in Ref. [四], where the complete result for the $\mathscr{O}\left(m_{e} \alpha^{7}\right)$ one-photon annihilation contribution has been derived. The perturbative corrections to HFS split into nonannihilation (radiative, radiative-recoil, and recoil corrections), one- and multiple-photon annihilation contributions. The nonannihilation and one-photon annihilation parts constitute about $47 \%$ and $32 \%$ of the second-order nonlogarithmic correction, respectively. Thus the one-photon annihilation contribution to the coefficient $D$ presumably gives a significant fraction of the total nonlogarithmic third-order correction.

In the following, we briefly outline our method of calculation. Perturbation theory of the positronium bound state has to be developed about the nonrelativistic Coulomb approximation rather than free electron and positron states. This can be done within the nonrelativistic effective field theory [30], which is a systematic way to separate the multiple scales characteristic to the bound-state problem. The bound-state dynamics involves three different scales: the hard scale of electron mass $m_{e}$, the soft scale of the bound-state three-momentum $\alpha m_{e}$, and the bound-state energy $\alpha^{2} m_{e}$. Integrating out the hard and soft degrees of freedom results in the potential nonrelativistic QED (pNRQED) [B]], an effective Schrödinger theory of a nonrelativistic electron-positron pair interacting with ultrasoft photons, which is a relevant framework for the calculation of the QED corrections to the positronium spectrum. We use dimensional regularization to deal with spurious divergences which appear in the process of scale separation. Systematic use of dimensional regularization [201, 32, [33] based on the asymptotic expansion approach [34, [35] is instrumental for the high-order analysis as it provides "built in" matching of the effective theory calculations to full QED.

The positronium HFS is given by the difference between the binding energy of the ortho and parapositronium states $\Delta v=E_{o}-E_{p}$. The leading order result can be written as $\Delta v^{L O}=$ $\left(\left[\frac{1}{3}\right]_{s c t}+\left[\frac{1}{4}\right]_{a n n}\right) \alpha^{4} m_{e}$, where nonannihilation (scattering) and one-photon annihilation contributions are given separately. By spin/parity conservation only the orthopositronium state is affected by the one-photon annihilation. The corresponding correction to the binding energy $E_{o}$ can be obtained by studying the threshold behavior of the vacuum polarization function $\Pi\left(q^{2}\right)$

$$
\left(q_{\mu} q_{v}-g_{\mu v} q^{2}\right) \Pi\left(q^{2}\right)=i \int d^{d} x e^{i q x}\left\langle 0\left|T j_{\mu}(x) j_{v}(0)\right| 0\right\rangle,
$$

where $j_{\mu}$ is the electromagnetic current, $q^{2}=\left(2 m_{e}+E\right)^{2}$ and $E$ is the energy counted from the threshold. Only one-particle irreducible contributions are retained on the right-hand side of Eq. (G) and the on-shell renormalization of the QED coupling constant requires $\Pi(0)=0$. The vacuum polarization function has bound-state poles at approximately Coulomb energies $E_{n}^{C}=-\alpha^{2} m_{e} /\left(4 n^{2}\right)$ with spin (orbital) angular momentum $S=1(l=0)$. Near the orthopositronium ground-state energy $E_{o}=E_{1}^{C}+\mathscr{O}\left(\alpha^{4}\right)$ it reads

$$
\lim _{E \rightarrow E_{o}^{\prime}} \Pi\left(q^{2}\right)=\frac{\alpha}{4 \pi} \frac{R_{o}}{E / E_{o}^{\prime}-1-i \varepsilon},
$$

where $E_{o}^{\prime}$ stands for $E_{o}$ without the total one-photon annihilation contribution. The pole position differs from the physical orthopositronium mass since the vacuum polarization function is defined 


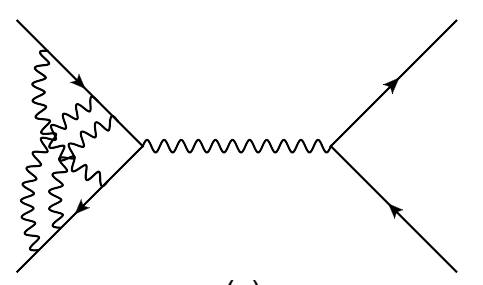

(a)

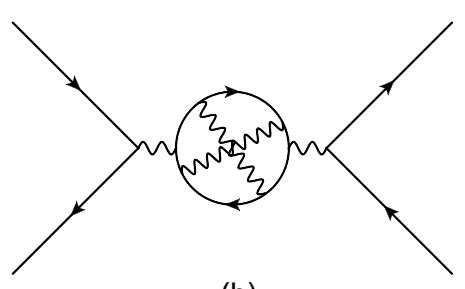

(b)

Figure 1: Three-loop Feynman diagrams contributing to (a) $R_{o}$ and (b) $P_{o}$.

as the one-particle irreducible contribution to the current correlator (G). By subtracting the pole one gets the regular part of the vacuum polarization function at $E=E_{o}^{\prime}$

$$
P_{o}=\lim _{E \rightarrow E_{o}^{\prime}}\left(e^{2} \Pi\left(q^{2}\right)-\frac{\alpha^{2} R_{o}}{E / E_{o}^{\prime}-1-i \varepsilon}\right) .
$$

Within the quantum-mechanical perturbation theory of pNRQED it is straightforward to derive the following expression for the one-photon annihilation contribution to the HFS

$$
\Delta_{\text {ann }}^{1-\gamma} v=\Delta_{\text {ann }}^{1-\gamma} E_{o}=\frac{\alpha^{4} m_{e}}{4} \frac{R_{o}}{1+P_{o}} .
$$

The factor $R_{o}$ in this equation has a natural interpretation: annihilation is a local process which probes the positronium wave function at the origin and the residue of Eq. (पD) defines this quantity in full QED beyond nonrelativistic quantum mechanics. On the other hand the factor $1 /\left(1+P_{o}\right)$ results from the Dyson resummation of the vacuum polarization corrections to the off-shell photon propagator in the annihilation amplitude. Eq. (Q) can be computed order by order in perturbation theory

$$
\Delta_{\text {ann }}^{1-\gamma} v=\frac{\alpha^{4} m_{e}}{4}\left[1+\sum_{n=1}\left(\frac{\alpha}{\pi}\right)^{n} h^{(n)}\right],
$$

where the coefficients $h^{(n)}$ are determined by the series $R_{o}=1+\sum_{n=1}\left(\frac{\alpha}{\pi}\right)^{n} r^{(n)}$ and $P_{o}=\sum_{n=1}\left(\frac{\alpha}{\pi}\right)^{n} p^{(n)}$ so that $h^{(1)}=r^{(1)}-p^{(1)}$ and so on. For the calculation of the third order corrections to the HFS we need all coefficients $r^{(n)}$ and $p^{(n)}$ up to $n=3$. Typical three-loop Feynman diagrams contributing to $R_{o}$ and $P_{o}$ are presented in Fig. 四.

The first-order coefficients get only a one-loop hard contribution $r^{(1)}=-4$ and $p^{(1)}=8 / 9$, which yields $h^{(1)}=-44 / 9$. In the second order the soft scale starts to contribute and one has to take into account an arbitrary number of Coulomb photon exchanges. The second-order correction to $R_{o}$ can be read off the QCD result for the photon-mediated heavy quarkonium production rate [B6, 137, [38, 139] by adopting the QED group factors $C_{F}=1, C_{A}=0, T_{F}=1$, as well as the number of the light (heavy) fermions $n_{l}=0\left(n_{h}=1\right)$

$$
r^{(2)}=\frac{527}{36}+\left(-\frac{2}{3} \ln \alpha-\frac{235}{72}+2 \ln 2\right) \pi^{2}-\zeta(3),
$$

where $\zeta(3)=1.20206 \ldots$ is a value of Riemann's zeta-function. By using the method outlined above we evaluate the second-order correction to $P_{o}$ with the result

$$
p^{(2)}=\frac{3}{4}+\left(-\ln \alpha+\frac{27}{16}-\frac{\ln 2}{2}\right) \frac{\pi^{2}}{2}-\frac{21}{8} \zeta(3) .
$$


This gives

$$
h^{(2)}=\frac{1477}{81}+\left(-\frac{\ln \alpha}{6}-\frac{1183}{288}+\frac{9}{4} \ln 2\right) \pi^{2}+\frac{13}{8} \zeta(3)
$$

in agreement with Ref. [ए]].

The third-order coefficients get contributions from all the scales present in the problem. By adjusting the QCD results [40, 4] we obtain the following expression

$$
\begin{aligned}
r^{(3)} & =-\frac{383}{18}+\left[-\frac{3}{2} \ln ^{2} \alpha+\left(-\frac{7}{90}+8 \ln 2\right) \ln \alpha\right. \\
& \left.-\frac{1019}{180}-4 \ln 2+\delta_{o}^{u s}\right] \pi^{2}+2 \zeta(3)-\frac{109}{864} \pi^{4}+2 c_{v 0}^{(3)} .
\end{aligned}
$$

Here $\delta_{o}^{u s}$ is an analog of the Bethe logarithm in hydrogen Lamb shift, which parametrizes the ultrasoft contribution [40]. It does not scale with the group factors and requires independent evaluation in the QED case, which gives $\delta_{o}^{u s}=18.8646(17)$ in agreement with [42]. The coefficient $c_{v 0}^{(3)}$ in Eq. (14) parametrizes the third-order hard contribution to the Wilson coefficient in the effective theory decomposition of the vector current $j=c_{\nu} \psi^{\dagger} \sigma \chi+\ldots$ in terms of the nonrelativistic electron and positron two-component Pauli spinors $\psi$ and $\chi$. The third-order term of the perturbative series $c_{v}=1+\sum_{n=1}^{\infty}\left(\frac{\alpha}{\pi}\right)^{n} c_{v}^{(n)}$ is given by the three-loop vertex diagrams (see e.g. Fig. $\mathbb{W}(\mathrm{a})$ ) evaluated at the threshold and has been recently computed in QCD [43]. The coefficients of the series are in general infrared divergent. These spurious divergences result from the scale separation in the effective theory framework and cancel out in the final result for physical observables. The value $c_{v 0}^{(3)}=35.76 \pm 0.53$ corresponds to the coefficient $c_{v}^{(3)}$ defined within the $\overline{\mathrm{MS}}$ subtraction scheme at the renormalization scale $\mu=m_{e}$. The logarithmic part of Eq. ([14) agrees with Ref. [44]]. The third-order term in Eq. (8) reads

$$
p^{(3)}=(2 \ln \alpha-3) \pi^{2}+p_{h 0}^{(3)}
$$

where the last term parametrizes the third-order hard contribution given by the three-loop vacuum polarization diagrams (see e.g. Fig. $($ (b)) evaluated at the threshold. As in the case of the vertex correction, this quantity is infrared divergent and the coefficient $p_{h 0}^{(3)}=0.16 \pm 0.04$ corresponds to the $\overline{\mathrm{MS}}$ subtraction scheme with $\mu=m_{e}$. By adding up all the relevant terms we get

$$
h^{(3)}=-\frac{3}{2} \pi^{2} \ln ^{2} \alpha+\left(-\frac{1181}{270}+8 \ln 2\right) \pi^{2} \ln \alpha+h_{0}^{(3)},
$$

where the nonlogarithmic part reads

$$
h_{0}^{(3)}=-\frac{49309}{1458}+\left(\frac{16573}{3240}-\frac{65}{9} \ln 2+\delta_{o}^{u s}\right) \pi^{2}-\frac{221}{18} \zeta(3)-\frac{109}{864} \pi^{4}+2 c_{v 0}^{(3)}-p_{h 0}^{(3)},
$$

or numerically $h_{0}^{(3)}=197.8 \pm 1.1$. In the above equation the scheme dependence of the coefficients $c_{v 0}^{(3)}$ and $p_{h 0}^{(3)}$ is canceled by the scheme dependence of the analytic part, which is also given in MS scheme. From the effective theory point of view the structure of the third-order logarithmic corrections in the one-photon annihilation contribution to the positronium HFS is identical to the 


\begin{tabular}{|c|c|c|c|}
\hline & $\ln ^{2} \alpha$ & $\ln \alpha$ & $D / \pi^{2}$ \\
\hline Positronium & $-\frac{3}{2}$ & $-\frac{62}{15}+\frac{68}{7} \ln 2 \approx 2.6001$ & $8.59(5)_{\text {ann }}^{1-\gamma}$ \\
Muonium & $-\frac{8}{3}$ & $-\frac{281}{180}+\frac{8}{3} \ln 2 \approx 0.2873$ & 16.233 \\
\hline
\end{tabular}

Table 1: The coefficients of $\alpha^{3} / \pi$ in perturbative series for positronium and muonium HFS.

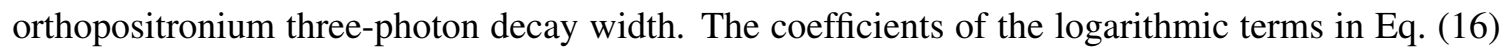
do agree with the series for the width [45, 46, 477] up to a substitution of the coefficient $\frac{A_{o}}{3} \rightarrow-\frac{2}{3} h^{(1)}$ in the interference term between the one-loop and the two-loop single-logarithmic corrections.

Finally for the third-order nonlogarithmic one-photon annihilation contribution to the HFS we obtain

$$
D_{\text {ann }}^{1-\gamma}=\frac{3}{7} h_{0}^{(3)}=84.8 \pm 0.5 .
$$

The coefficients of the third-order corrections to HFS in positronium and muonium atom [27] are compared in Table $\square$. It is interesting to note that the ultrasoft contribution due to $\delta_{o}^{u s}$ approximates the complete result (18) with 5\% accuracy. The nonannihilation contribution includes a similar term and we may speculate that it is also dominated by the ultrasoft contribution. This does not seem implausible since the fully relativistic corrections from the hard scale are known to usually be suppressed. For example, the pure radiative corrections to the HFS related to the electron anomalous magnetic moment $a_{e}, \Delta_{a_{e}} v=\left(\alpha^{4} m_{e} / 4\right)\left[\left(1+a_{e}\right)^{2}-1\right]$, gives only a tiny contribution $D_{a_{e}}=1.16229 \ldots$, where we used the two and three-loop result for $a_{e}$ [ए], [8]]. Another example is a recently computed nonannihilation correction due to closed electron loops [49, 50], which is also numerically suppressed. In this case the nonannihilation contribution would be given by $D_{s c t} \approx \frac{4 \pi^{2}}{7} \delta_{o}^{u s} \approx 106$, which slightly exceeds the one-photon annihilation contribution (18) in full analogy with the structure of the second-order corrections. Then we get an estimate $D \approx 191$, which is close to the muonium result.

To summarize, the calculation of the $\mathscr{O}\left(\alpha^{7} m_{e}\right)$ one-photon annihilation contribution to the positronium HFS [U] provides the first nontrivial third-order QED result in positronium spectroscopy beyond the logarithmic approximation. This opens a prospect of advancing the theoretical analysis of positronium to a completely different level of precision. Numerically the new contribution increases the QED prediction by $217 \pm 1 \mathrm{kHz}$. Our final prediction for the positronium HFS including the $\mathscr{O}\left(\alpha^{7} m_{e}\right)$ one-photon annihilation term reads

$$
\Delta v^{\text {th }}=203.39191(22) \mathrm{GHz},
$$

where the error due to the missing part of the $\mathscr{O}\left(\alpha^{7} m_{e}\right)$ corrections is given by the size of the evaluated one-photon annihilation contribution (118).

\section{References}

[1] M. Baker, P. Marquard, A. Penin, J. Piclum and M. Steinhauser, Phys. Rev. Lett. 112, 120407 (2014).

[2] S. L. Glashow, Phys. Lett. B 167 (1986) 35.

[3] A. P. Mills, Jr. and G. H. Bearman, Phys. Rev. Lett. 34, 246 (1975). A. P. Mills, Phys. Rev. A 27, 262 (1983). 
[4] M. W. Ritter, P. O. Egan, V. W. Hughes, and K. A. Woodle, Phys. Rev. A 30, 1331 (1984).

[5] A. Ishida, T. Namba, S. Asai, T. Kobayashi, H. Saito, M. Yoshida, K. Tanaka and A. Yamamoto, arXiv:1310.6923 [hep-ex].

[6] J. Pirenne, Arch. Sci. Phys. Nat. 29, 265 (1947).

[7] V. B. Berestetski and L. D. Landau, Zh. Eksp. Teor. Fiz. (USSR) 19, 673 (1949).

[8] R. A. Ferrell, Phys. Rev. 84, 858 (1951).

[9] R. Karplus and A. Klein, Phys. Rev. 87, 848 (1952).

[10] S. J. Brodsky and G. W. Erickson, Phys. Rev. 148, 26 (1966).

[11] R. Barbieri, J. A. Mignaco and E. Remiddi, Nuovo Cim. A 11, 824 (1972).

[12] G. T. Bodwin and D. R. Yennie, Phys. Rept. 43, 267 (1978).

[13] W. E. Caswell and G. P. Lepage, Phys. Rev. A 20, 36 (1979).

[14] J. R. Sapirstein, E. A. Terray and D. R. Yennie, Phys. Rev. D 29, 2290 (1984).

[15] G. S. Adkins, M. H. T. Bui and D. Zhu, Phys. Rev. A 37, 4071 (1988).

[16] G. S. Adkins, Y. M. Aksu and M. H. T. Bui, Phys. Rev. A 47, 2640 (1993).

[17] A. H. Hoang, P. Labelle and S. M. Zebarjad, Phys. Rev. Lett. 79, 3387 (1997); Phys. Rev. A 62, 012109 (2000).

[18] K. Pachucki, Phys. Rev. Lett. 79, 4120 (1997); Phys. Rev. A 56, 297 (1997).

[19] K. Pachucki and S. G. Karshenboim, Phys. Rev. Lett. 80, 2101 (1998).

[20] A. Czarnecki, K. Melnikov and A. Yelkhovsky, Phys. Rev. Lett. 82, 311 (1999); Phys. Rev. A 59, 4316 (1999).

[21] G. S. Adkins and J. Sapirstein, Phys. Rev. A 58, 3552 (1998) [Erratum ibid. 61069902 (2000)].

[22] A. P. Burichenko, hep-ph/0004063.

[23] S. G. Karshenboim, Zh. Eksp. Teor. Fiz. 103, 1105 (1993) [Sov. Phys. JETP 76, 541 (1993)].

[24] R. J. Hill, Phys. Rev. Lett. 86, 3280 (2001).

[25] K. Melnikov and A. Yelkhovsky, Phys. Rev. Lett. 86, 1498 (2001).

[26] B. A. Kniehl and A. A. Penin, Phys. Rev. Lett. 85, 5094 (2000).

[27] M. Nio and T. Kinoshita, Phys. Rev. D 55, 7267 (1997), and references therein.

[28] T. Yamazaki, A. Miyazaki, T. Suehara, T. Namba, S. Asai, T. Kobayashi, H. Saito and I. Ogawa et al., Phys. Rev. Lett. 108, 253401 (2012).

[29] D. B. Cassidy, T. H. Hisakado, H. W. K. Tom and A. P. Mills, Phys. Rev. Lett. 109, 073401 (2012).

[30] W. E. Caswell and G. P. Lepage, Phys. Lett. B 167, 437 (1986).

[31] A. Pineda and J. Soto, Nucl. Phys. Proc. Suppl. 64, 428 (1998).

[32] A. Pineda and J. Soto, Phys. Lett. B 420, 391 (1998).

[33] B. A. Kniehl, A. A. Penin, V. A. Smirnov and M. Steinhauser, Nucl. Phys. B 635, 357 (2002).

[34] M. Beneke and V. A. Smirnov, Nucl. Phys. B 522, 321 (1998). 
[35] V. A. Smirnov, Applied asymptotic expansions in momenta and masses, Springer Tracts Mod. Phys. 177 (2002) 1.

[36] K. Melnikov and A. Yelkhovsky, Phys. Rev. D 59 (1999) 114009.

[37] A. A. Penin and A. A. Pivovarov, Nucl. Phys. B 549, 217 (1999).

[38] A. Czarnecki and K. Melnikov, Phys. Rev. Lett. 80, 2531 (1998).

[39] M. Beneke, A. Signer, and V. A. Smirnov, Phys. Rev. Lett. 80, 2535 (1998).

[40] M. Beneke, Y. Kiyo and A. A. Penin, Phys. Lett. B 653, 53 (2007).

[41] M. Beneke, Y. Kiyo and K. Schuller, Phys. Lett. B 658, 222 (2008).

[42] S.R. Marcu, Master's Thesis in Physics, University of Alberta (Edmonton, 2011).

[43] P. Marquard, J. H. Piclum, D. Seidel and M. Steinhauser, Phys. Rev. D 89, 034027 (2014).

[44] B. A. Kniehl, A. A. Penin, V. A. Smirnov and M. Steinhauser, Phys. Rev. Lett. 90, 212001 (2003).

[45] B. A. Kniehl and A. A. Penin, Phys. Rev. Lett. 85, 1210 (2000) [Erratum ibid. 85, 3065 (2000)].

[46] R. J. Hill and G. P. Lepage, Phys. Rev. D 62, 111301 (2000).

[47] K. Melnikov and A. Yelkhovsky, Phys. Rev. D 62, 116003 (2000).

[48] S. Laporta and E. Remiddi, Phys. Lett. B 379, 283 (1996).

[49] G. S. Adkins and R. N. Fell, Phys. Rev. A 89, 052518 (2014).

[50] M. I. Eides and V. A. Shelyuto, arXiv:1403.7947 [hep-ph]. 